\title{
Diagnostic and Management Tool for Monitoring Patients Implanted with a Shape-Changing Corneal Inlay
}

\author{
Ralph Chu ${ }^{a}$ Briana R. Lee ${ }^{b}$ \\ ${ }^{a}$ Chu Vision Institute, Bloomington, MN, USA; ${ }^{b}$ ReVision Optics Inc., Lake Forest, CA, USA
}

\section{Keywords}

Corneal inlay $\cdot$ Pentacam $\cdot$ Haze $\cdot$ Raindrop Near Vision Inlay

\begin{abstract}
We report two cases that underwent corneal inlay implantation under a femtosecond flap. The first case had no complications during the postoperative period. Pentacam maps remained stable over time: corneal densitometry $(C D)$ values had less than 2 units of change across visits up to 3 years. Axial/sagittal topography maps showed a stable $K_{\max }(\sim 47$ D), i.e., less than $1 \mathrm{D}$ change between visits. The second case developed haze at 8 months postoperatively. CD increased by more than 4 units from 3 to $8 \mathrm{M}$ and $\mathrm{K}_{\max }$ increased by $2.6 \mathrm{D}$. The patient was prescribed steroids and 1 month later the haze regressed, resulting in pre-haze $C D$ and $K_{\max }$ values, similar to the ones at 3 months.

(C) 2018 The Author(s) Published by S. Karger AG, Basel
\end{abstract}

\section{Introduction}

Corneal inlays are a new alternative for the surgical correction of presbyopia. The Raindrop Near Vision Inlay (ReVision Optics, Inc., Lake Forest, CA, USA) is made of a biocompatible hydrogel material [1] that is designed to reshape the cornea to improve near vision. In the US FDA clinical trial, near vision was improved by 5 lines, and intermediate vision improved by $\sim 2.5$ lines at 24 months postoperatively [2]. During the course of the study, the most frequent complication reported was haze in the area where the inlay is lo- 


\section{Case Reports in Ophthalmology}

Chu and Lee: Diagnostic and Management Tool for Monitoring Patients Implanted with a Shape-Changing Corneal Inlay

cated; its incidence decreased when the inlay was implanted at a 30\% depth of the central corneal thickness (CCT). Corneal haze affects the transmission of light through the cornea, resulting in backward scattering of light, and is associated with decreased distance visual acuities [3]. Clinically, haze is evaluated through a slit lamp examination, in which an observer documents subjectively. While changes to visual acuity and slit lamp analysis can assess corneal health after surgery, these methods are subjective and have limitations when tracking small variations (progression/regression) over time. The development of an objective method to monitor patients with haze then becomes a critical tool for diagnosing haze, tracking its progression and determining if intervention is necessary.

The rotating Scheimpflug camera provides a broad set of applications including topography, pachymetry, and anterior chamber depth (Pentacam, Oculus Optikgeräte GmbH). It also composes a map of the amount of backscattered light (corneal densitometry [CD], included as part of the software's refractive package), providing precise and reproducible information regarding corneal clarity. The densitometry measurements are expressed in grayscale units (GSU) and range from 0 (minimum light scatter, 100\% transparency) to 100 (maximum light scatter, $0 \%$ transparency) depending on the degree of corneal light scatter. It analyzes the cornea within a diameter of $12 \mathrm{~mm}$ (in 4 concentric zones and at 3 depths). Total CD values were noted in terms of zone and depth [4]. Because the diameter of the Raindrop Near Vision Inlay is $2 \mathrm{~mm}$, we focused on the 0 - to 2-mm annulus value.

This report describes two cases of emmetropic presbyopes that were implanted with the Raindrop Inlay and followed using Scheimpflug imaging and vision assessment over a 36-month postoperative period.

\section{Case Reports}

\section{Case 1}

Before surgery, before implantation with the Raindrop Inlay, the patient had an uncorrected near visual acuity (UNVA) of 20/50 and uncorrected distance visual acuity (UDVA) of $20 / 25$. The inlay was implanted in the nondominant eye under a $173-\mu \mathrm{m}$ flap $(\sim 33 \%$ CCT), centered over the light-constricted pupil. Her early postoperative period was unremarkable; at her 1-year visit, the patient achieved 20/20 UDVA and UNVA. In topography, central corneal steepening was shown immediately after implantation and was stable at all visits using the axial/sagittal map display (Fig. 1, top). Difference maps showed a 3.2-D steepening in $\mathrm{K}_{\max }$ from the preoperative period (see the Pentacam manual for details on $\mathrm{K}_{\max }$ ). Slit lamp examination revealed a clear central cornea with only a slight ring visible around the edge of the inlay. Using densitometry, the value within $0-2 \mathrm{~mm}$ was $17.7 \mathrm{GSU}$ at the preoperative period and at 12 months, it was 19.1 GSU. The opacity map on the left at the 150 - to $180-\mu \mathrm{m}$ region showed some backscatter around the periphery of the inlay. This ring around the inlay is normal and the $\mathrm{CD}$ value will increase by a few units when compared to the virgin cornea.

At 36 months, this patient's uncorrected distance and near visual acuities were all still $20 / 20 . K_{\max }$ change was $\sim 1 \mathrm{D}$ from 12 to 36 months. The CD value was stable at $17.2 \mathrm{GSU}$ (Fig. 1, bottom). Overall, stability was observed up to 36 months.

\section{Case 2}

The second case had a preoperative UNVA of 20/80 and UDVA of 20/20. The inlay was also implanted in the nondominant eye under a 174- $\mu \mathrm{m}$ flap ( $\sim 33 \% \mathrm{CCT})$, centered over the 
pupil. At 3 months, the patient was stable, achieving uncorrected distance and near visual acuities of 20/20; the central corneal steepening was adequate (+3.1 D increase in $K_{\max }$ ). The CD value was $18.8 \mathrm{GSU}$. At 6 months, the slit lamp examination revealed some peripheral haze; visual acuities remained $20 / 20$ at both distances, but the cornea steepened ( $\mathrm{K}_{\max }$ increased by $1.4 \mathrm{D}$ ) and the CD value went up to $20.5 \mathrm{GSU}$. No treatment was prescribed. Two months later, the patient returned with decreased UDVA (20/50) associated with a slight myopic shift of $-0.25 \mathrm{D}$; the slit lamp examination showed central corneal haze progression, now encroaching toward the center of the inlay and topography showed a steepening at the inlay interface (Fig. 2), with further increase in $\mathrm{K}_{\max }$ by another +1.2 D. The CD value also increased to 23.0 GSU (Fig. 3, top). A Durezol (Novartis) taper for 1 month was prescribed. At the next visit, distance visual acuity improved to $20 / 25$, and near visual acuity remained at 20/20. $\mathrm{K}_{\max }$ regressed to $46.8 \mathrm{D}(-3.1 \mathrm{D})$, similar to the 3-month $\mathrm{K}_{\max }$. The slit lamp examination showed minimal haze at the center of the inlay and the CD value decreased to 19.3 (Fig. 3, bottom). The patient had stable visual acuities, refraction, $\mathrm{K}_{\max }$, and CD values following treatment, similar to what they were shortly after implantation.

\section{Discussion}

Since Barraquer first introduced the idea of corneal implants for the correction of ametropias, corneal haze (and its complications) has been the main drawback of these devices. New materials and a better understanding of the corneal wound healing response (including ideal depth placement within the stroma) have reduced the rates of complications and allowed the recent FDA approval of two inlays indicated for the correction of presbyopia.

Corneal haze can be attributed to increased activation of keratocytes, increased disorganization and quantity of collagen fibrils, and a change in extracellular matrix composition resulting in more backscattering of light which translates into some loss of corneal transparency. While the gold standard for corneal clarity evaluation is to use slit lamp biomicroscopy, this is a subjective method and there may be variations from one observer to another and from visit to visit. Utilization of anterior segment optical coherence tomography or confocal imaging gives finer resolution of the corneal stroma [5], yet access to these diagnostic devices are limited and not widely adopted in a clinical setting. Scheimpflug optical devices, on the other hand, are more widely available in refractive clinics.

The addition of CD software makes this machine a very valuable tool when evaluating inlay patients. Scheimpflug imaging provides a more objective and reproducible method by utilizing transmission of light through the cornea to assess opacity. Increased backscatter can be easily detected using this device even in corneas that have been considered clinically clear [6]. In addition, this noninvasive measurement can be used to optically assess corneal topography. This evaluation of corneal tissue provides valid and reproducible data with minimal examiner experience $[7,8]$.

The two cases that we are reporting show our experience monitoring patients implanted with a transparent corneal inlay using this technology. Our recommendation for following patients with a corneal inlay is to obtain a scan at every visit in association with a basic examination (slit lamp, visual acuities, refraction); a normal visit for a corneal inlay patient after surgery should include no significant changes in visual acuity, refraction, and slit lamp evaluation. The same is seen with corneal densitometry and topography changes utilizing Scheimpflug imaging. CD values and topography $\mathrm{K}$ changes should show minimal variation from visit to visit. While myself and other surgeons have experienced increased keratometry 
values and decreased distance vision when corneal haze encroaches toward the center of the inlay, calculation of correlation is limited due to a small sample size of such cases. In addition, Pentacam imaging is not always reliable at capturing images when patients have frequent blinking, photophobia, or excessive tearing [9]. Additionally, when there is significant haze in the cornea, the gating across the cornea may not be reliable - the gating may be below the epithelium, where haze starts, resulting in topography reading such that the cornea appears to be flattening.

This is the first report, to our knowledge, that objectively assesses corneal clarity and topographical changes in patients implanted with a corneal inlay. This automated tool allows for rapid measurements that will benefit both the patient and health care practitioner when it comes to monitoring corneal health as well as detecting and treating complications.

\section{Acknowledgements}

This work was funded by ReVision Optics, Inc.

\section{Statement of Ethics}

All patients included in this paper were part of the US FDA IDE clinical investigation (NCT01373580), which received Institutional Review Board approval and adhered to the Declaration of Helsinki.

\section{Disclosure Statement}

Dr. Chu is a consultant and clinical investigator to ReVision Optics, Inc. Dr. Lee is an employee of ReVision Optics, Inc. with financial and proprietary interests.

\section{References}

1 Pinsky PM: Three-dimensional modeling of metabolic species transport in the cornea with a hydrogel intrastromal inlay. Invest Ophthalmol Vis Sci 2014;55:3093-3106.

2 ReVision Optics: Raindrop Near Vision Inlay Professional Use Information. Lake Forest, ReVision Optics, Inc, 2017.

3 Whitman J, et al: Treatment of presbyopia in emmetropes using a shape-changing corneal inlay: oneyear clinical outcomes. Ophthalmology 2016;123:466-475.

4 Ni Dhubhghaill S, et al: Normative values for corneal densitometry analysis by Scheimpflug optical assessment. Invest Ophthalmol Vis Sci 2014;55:162-168.

5 Hillenaar T, et al: Normative database for corneal backscatter analysis by in vivo confocal microscopy. Invest Ophthalmol Vis Sci 2011;52:7274-7281.

-6 Patel SV, McLaren JW, Hodge DO, Bourne WM: The effect of corneal light scatter on vision after penetrating keratoplasty. Am J Ophthalmol 2008;146:913-919.

-7 Shankar H, Taranath D, Santhirathelagan CT, Pesudovs K: Repeatability of corneal first-surface wavefront aberrations measured with Pentacam corneal topography. J Cataract Refract Surg 2008;34:727-734.

-8 Shankar H, Taranath D, Santhirathelagan CT, Pesudovs K: Anterior segment biometry with the Pentacam: comprehensive assessment of repeatability of automated measurements. J Cataract Refract Surg 2008;34:103-113.

-9 Otri AM, Fares U, Al-Aqaba MA, Dua HS: Corneal densitometry as an indicator of corneal health. Ophthalmology 2012;119:501-508. 


\section{Case Reports in Ophthalmology}

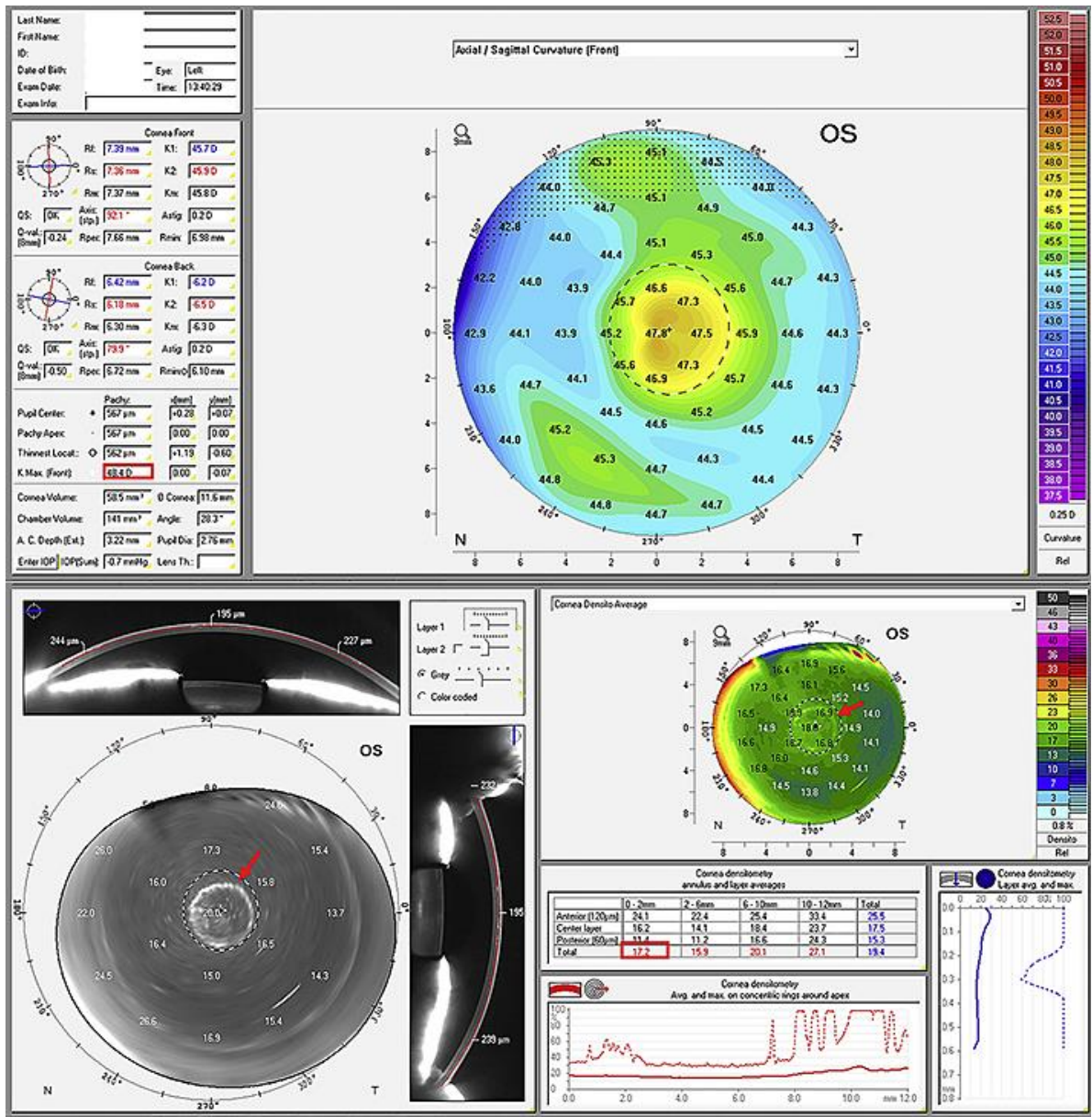

Fig. 1. Top: axial/sagittal topography map showing central corneal steepening after inlay implantation. $\mathrm{K}_{\max }$ value is boxed in red. Bottom: corneal densitometry (CD) at 36 months postoperatively showing a slight ring of corneal opacity at the inlay periphery (arrow), and the CD value in red.

Chu and Lee: Diagnostic and Management Tool for Monitoring Patients Implanted with a Shape-Changing Corneal Inlay 
Chu and Lee: Diagnostic and Management Tool for Monitoring Patients Implanted with a Shape-Changing Corneal Inlay

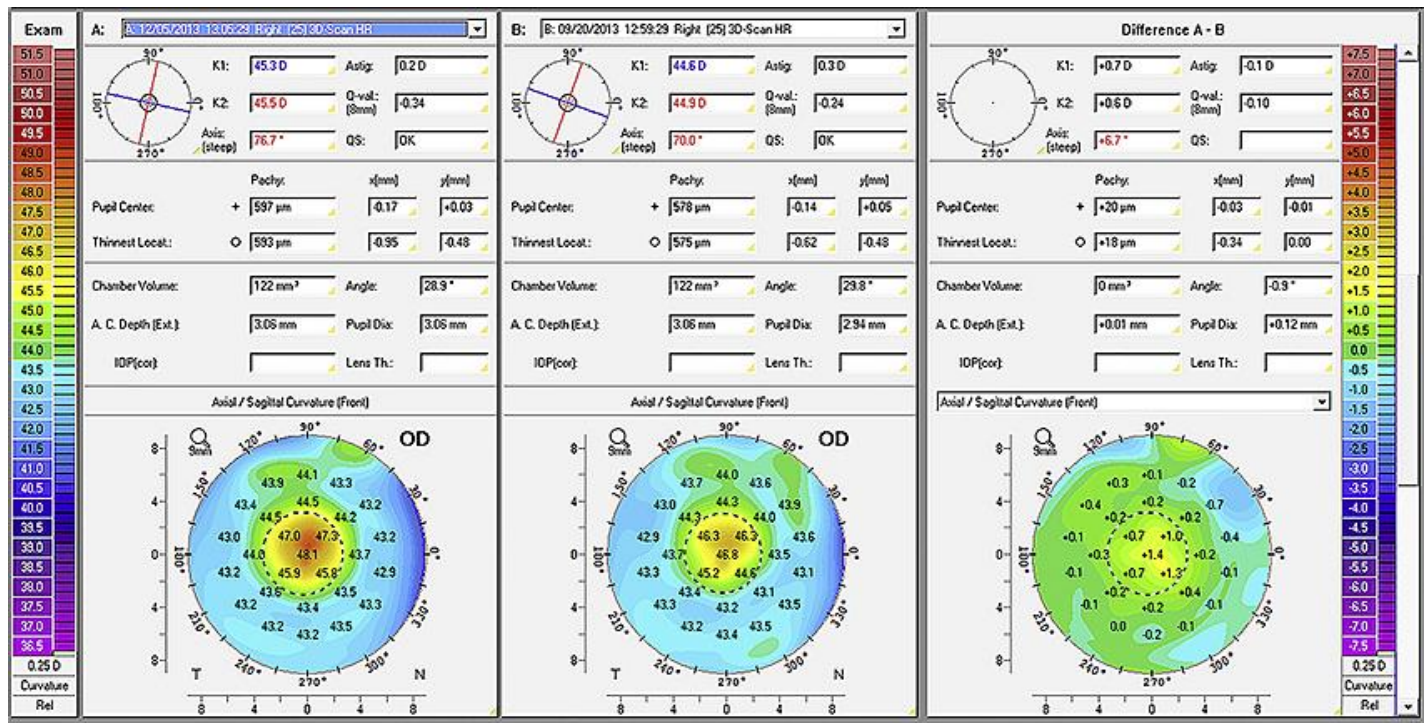

Fig. 2. Axial/sagittal curvature maps showing additional steepening of the central cornea over time and represented as a difference map. 


\section{Case Reports in Ophthalmology}
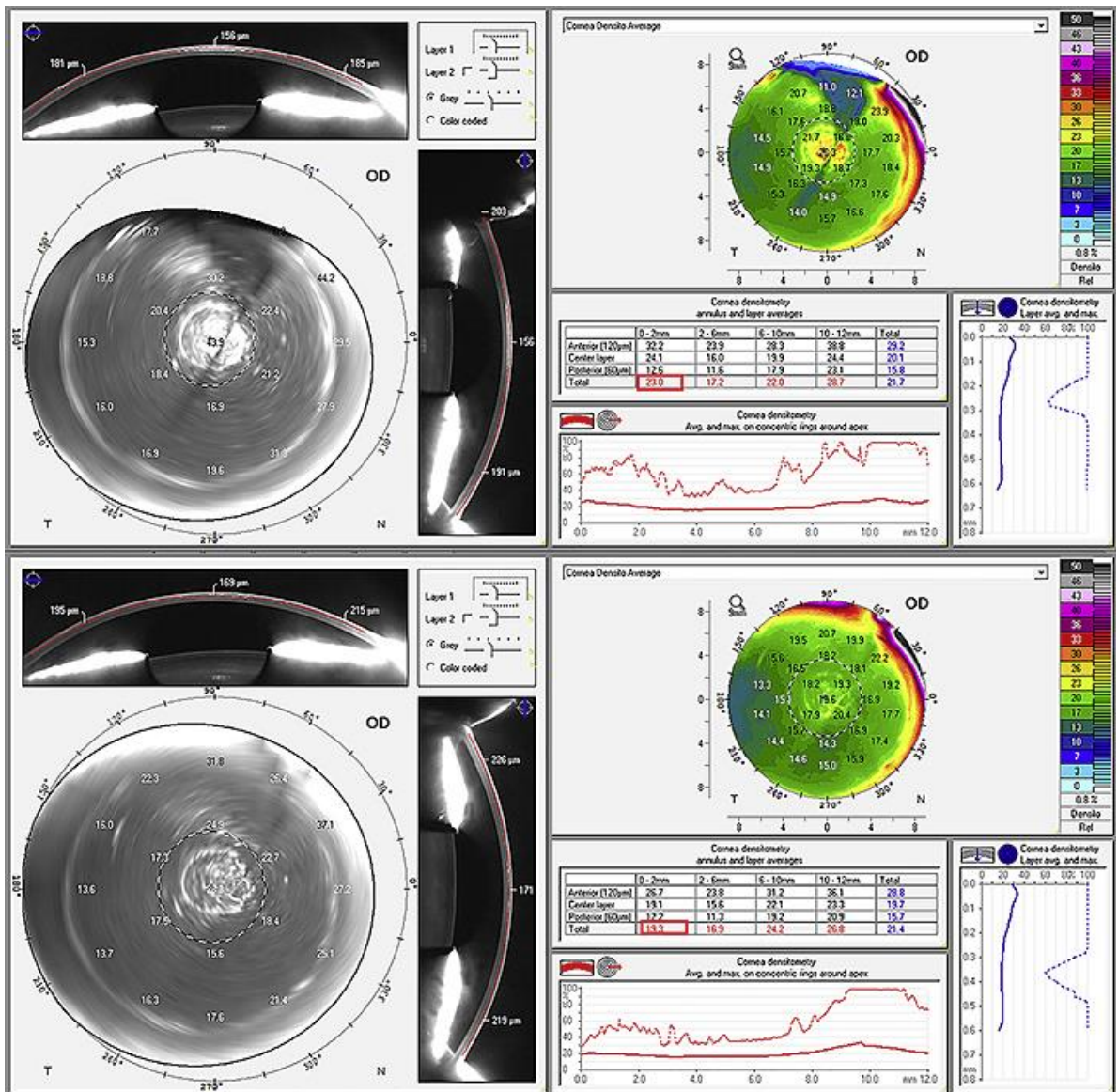

Fig. 3. Top: corneal densitometry (CD) showing central corneal haze and increased densitometry values in the 0 - to 2-mm zone with $\mathrm{CD}$ value in red. Bottom: regressed values after steroid treatment with $\mathrm{CD}$ value in red. 